\title{
ЭМЕРГЕТИЧЕСКИЙ ПОДХОД К АНАЛИЗУ ПРОИЗВОДСТВЕННОЙ СИСТЕМЫ НА ПРИМЕРЕ ПРОИЗВОДСТВА ЦЕМЕНТА
}

\section{AN EMERGING APPROACH TO THE ANALYSIS OF A PRODUCTION SYSTEM USING THE EXAMPLE OF CEMENT PRODUCTION}

\section{E. Romanova}

Summary. The paper provides an emerging analysis of information about the production system. A significant environmental load has been identified, and the results of calculations of alternative technologies are presented. The proposed technique can be used to implement a system for modeling production systems based on the emerging approach.

Keywords: emergia, production system, information analysis.

\author{
Романова Елена Юрьевна \\ К.п.н., Российский государственный социальный \\ университет (2. Москва) \\ klenar2001@mail.ru
}

Аннотация. В работе проведен эмергетический анализ информации о производственной системы. Выявлена значительная экологическая нагрузка, приведены результаты расчетов альтернативных вариантов технологий. Предлагаемая методика может быть использована для реализации системы моделирования производственных систем на основе эмергетического подхода.

Ключевые слова: эмергия, производственная система, анализ информации. $\boldsymbol{9}$ мергия определяется как сумма всех прямых и косвенных затрат энергии, используемых в процессе производства продукта или услуг ([1, 2]). Солнечная энергия является основным источником, который питает все процессы и циклы на Земле. Эмергия, а также показатели, конструируемые на ее основе, не являются эмпирическим свойством объекта, а являются оценкой воплощенной энергии на основе соответствующего набора эмпирических данных из систем, лежащие в основе объекта или процесса, а также правил и теоретических допущений и, следовательно, не могут быть непосредственно измерены.

Чтобы оценить эмергию солнечного излучения для конкретного ресурса, продукта или услуги, нужно отследить все потоки ресурсов, энергии, которые были использованы для его получения. Эти входные потоки, в свою очередь, также должны быть выражены в единицах солнечной энергии. Заметим, что оценка эмергии, проводится в условиях числовой неопределенности, которая возникает на всех этапах и из всех источников, используемых в процессе оценки. Эмергия измеряется в солнечно-эквивалентных джоулях (solar-equivalent joules, sej), обозначаемых эмДж.

Сбор информации о компонентах производственной системы и учет их взаимодействия позволяет рассчитать генерируемую эмергию. Эмергетический анализ стано- вится инструментом для анализа экологических и экономических последствия производственной системы.

Метод эмергетического анализа включает следующие этапы:

- построение диаграммы системы, учитывающей все взаимодействия внутри системы (системная диаграмма). Компоненты могут быть природного происхождения (возобновляемые и невозобновляемые природные ресурсы) или экономического происхождения (материалы, услуги и денежный оборот).

- заполнение таблицы эмергии используемых ресурсов и расчет трансформации эмергии этих ресурсов.

- расчет индексов эмергии ([1, 2]), что позволяет проводить анализ. Основные показатели эмергетического анализа и предлагаемая интерпретация представлены в Таблице 1.

В работе [3] с помощью эмергетического анализа изучалась целесообразность культивации в Бразилии сахарного тростника как сырья для производства этанола - одного из источников энергии. В работе [4] в качестве источников энергии в производственной системе сахарного тростника рассматривались: дождь; ветер; солнце; сельскохозяйственные угодия; промышленные и городские отходы; инфраструктура; механизирован- 
Таблица 1. Некоторые индексы эмергетического анализа

\begin{tabular}{|c|c|c|}
\hline Индекс & Формула, интерпретация & Ед. измерения \\
\hline $\begin{array}{l}\text { Суммарная эмергия, Total emergy per } \\
\text { year (Y) }\end{array}$ & Y=R + N + F Суммарная эмергия (экспорт не учитывается) & эмДж \\
\hline $\begin{array}{l}\text { Эмергия на одного человека, } \\
\text { Emergy per capita (EP) }\end{array}$ & $\begin{array}{l}\text { Y/Population } \\
\text { Отражает качество жизни населения }\end{array}$ & эмДж/чел \\
\hline $\begin{array}{l}\text { Солнечное преобразование, } \\
\text { затратность, transformity (Tr) }\end{array}$ & $\begin{array}{l}\text { Рассчитывается как сумма эмергий всех входящих потоков, деленная } \\
\text { на энергию продукта. Чем больше значение трансформации, тем более } \\
\text { важным является ресурс для экосистем и людей. }\end{array}$ & эмДж Дж ${ }^{-1}$ \\
\hline $\begin{array}{l}\text { Процент возобновляемости, } \\
\text { Renewability percentage (R\%) }\end{array}$ & $\begin{array}{l}\text { \%R=R/Y } \\
\text { Указывает, какая часть эмергии, используемой в производстве, поступает } \\
\text { от возобновляемых источников. Чем больше значение, тем больше } \\
\text { устойчивость системы. }\end{array}$ & $\%$ \\
\hline $\begin{array}{l}\text { Коэффициент эмергетической } \\
\text { отдачи, Emergy yield ratio (EYR) }\end{array}$ & $\begin{array}{l}\text { EYR=Y/F } \\
\text { Взаимосвязь между эмергией процесса и суммой эмергий всех потоков. } \\
\text { Указывает, возвращает ли процесс больше в экономику, чем расходует } \\
\text { в производство, является показателем того, насколько инвестиции } \\
\text { позволяют процессу использовать местные ресурсы для дальнейшего вклада } \\
\text { в экономику. }\end{array}$ & $\%$ \\
\hline $\begin{array}{l}\text { Коэффициент инвестирования } \\
\text { эмергии, Emergy investment ratio } \\
\text { (EIR) }\end{array}$ & $\begin{array}{l}\text { EIR=F/I } \\
\text { Отношение эмергии производительных сил, экономических (F) ресурсов, } \\
\text { которые были задействованы, к эмергии природных (возобновляемых } \\
\text { и невозобновляемых) ресурсов, которые предлагаются местной средой. } \\
\text { Это соотношение отражает интенсивность экономического развития } \\
\text { и степень нагрузки на окружающую среду. Чем больше значение отношения, } \\
\text { тем больше экономическое развитие. Кроме того, это отношение можно } \\
\text { использовать для сравнения различных систем, чтобы определить, какая } \\
\text { из них более экономически конкурентоспособна. Отношение может } \\
\text { отражать нагрузку, оказываемую экономической деятельностью на местную } \\
\text { экосистему. }\end{array}$ & $\%$ \\
\hline $\begin{array}{l}\text { Коэффициент экологической } \\
\text { нагрузки, Environmental load ratio } \\
\text { (ELR) }\end{array}$ & $\begin{array}{l}\text { ELR=(F+N)/R } \\
\text { Отношение суммы эмергий производительных сил (F) и невозобновляемых } \\
\text { природных ресурсов (N), к эмергии возобновляемым ресурсов (I). Чем выше } \\
\text { этот коэффициент, тем выше воздействие системы на окружающую среду. } \\
\end{array}$ & $\%$ \\
\hline $\begin{array}{l}\text { Коэффициент эмергетической } \\
\text { устойчивости, Emergy sustainability } \\
\text { index (ESI) }\end{array}$ & $\begin{array}{l}\text { ESI=EYR / ELR Oн выражает комплексное воздействие окружающей среды } \\
\text { и экономики на оцениваемую систему }\end{array}$ & $\%$ \\
\hline $\begin{array}{l}\text { Коэффициент пересчета эмергии, } \\
\text { Emergy exchange ratio (EER) }\end{array}$ & $\begin{array}{l}\text { EER=Eprod/Emoney } \\
\text { Отношение эмергии продукта, к количеству эмергии денег, полученных } \\
\text { от продажи продукта. Экономика подвергается риску, если продает больше } \\
\text { эмергии в виде продуктов, чем получает в денежной форме }\end{array}$ & $\%$ \\
\hline $\begin{array}{l}\text { Денежный коэффициент эмиссии, } \\
\text { Emergy money ratio (EMR) }\end{array}$ & Y/GDP Отношение суммарной эмергии к ВBП & эмДж/д.е. \\
\hline
\end{tabular}

ные операции; физический труд; деньги и административные ресурсы; почва; воды; биоразнообразия и остатки; материалы; услуги; цены на сахарный тростник, сахар и алкоголь.

В работе [5] предлагается включить фактор возобновляемости в учет эмергии материалов (M) и услуг (S). Соответственно модифицируются индексы EYR, EIR, ELR, уточняется их интерпретация. Подчеркивается важность такого методологического подхода при анализе устойчивости локальной экономики.
В статье [6] проводится анализ локальной экономики. Отмечается, динамика индексов ED и EPC указывает на устойчивое развитие экономики и повышение благосостояния населения, за счет увеличения доли невозбновляемых ресурсов в сельскохозяйственном производстве (большое значение индекса EIR). Значение индекса EYR и EIR говорит о повышении нагрузки на окружающую среду без существенного увеличения производительности. Использованный метод анализа на основе сценариев устойчивости показал, что увеличение доли возобновляемых ресурсов в сельском хозяйстве приве- 


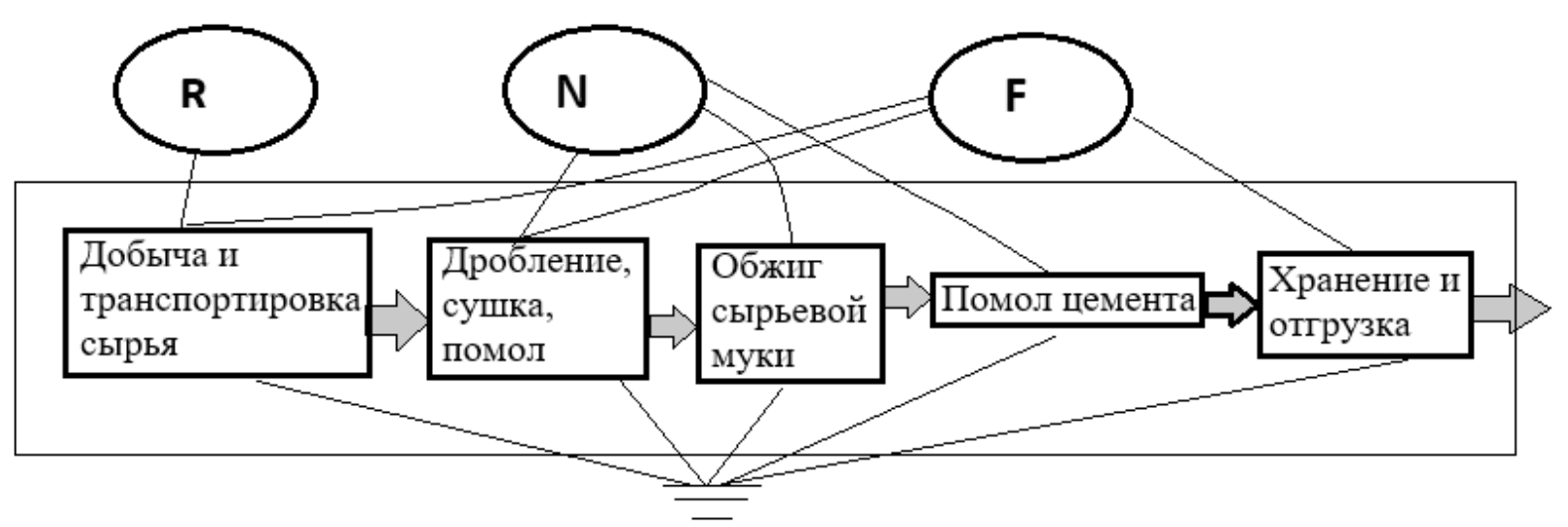

Рис. 1. Диаграмма системы производства цемента

дет к синхронному снижению нагрузки на окружающую среду и экономики.

В статье [7] приведен очень хороший обзор предшествующих работ по эмергетическому анализу. Авторы предлагают дополнить эмергетический анализ IPAT моделью. IPAТ модель представляет собой уравнение, связывающее воздействие на окружающую среду (I) с населением (Р), благосостоянием (А) и технологиями (Т), позволяет изучить взаимосвязь между экономическим ростом, ресурсами и окружающей средой.

Корреляционные связи между различными эмергетическими индексами и экономическим развитием стран исследованы на основе базы данных NEAD в работе [8].

В работах $[10,11]$ эмергетический подход применяется как основа для интегрированного изучения гео-, био- и ноосферных процессов, при этом подчеркивается необходимость разработки методик адаптации эмергетического подхода к уровням географических локаций и отдельных предприятий.

В данной работе за основу предмета эмергетического исследования взяты базовые производственные и географические характеристики производства цемента на примере AO «ХайдельбергЦемент Волга» (г. Вольск Саратовской области) без учета новшеств, которые применяются заводом для повышения конкурентноспособности.

Цементная промышленность как вид тяжелой промышленности оказывает разрушительное воздействие на окружающую среду. Так, при производстве 1 миллиона тонн цемента в год выбросы в атмосферу составляют: 6,65 тыс. тонн диоксида серы (SO2), 5,06 тыс. тонн оксидов азота $\left(\mathrm{NO}_{2}\right)$ и 6,57 тыс. тонн твердых частиц [12], промышленных сточные вод 9,54 тыс. тонн, твердых отходов17,06 тыс. тонн. Рассматриваемый завод внедряет технологические процессы, снижающие экологические и энергетические нагрузки (такие, как «сухой» способ, использование печи с декарбонизатором и одноветьевым пятиступенчатым циклонным теплообменником).

На Рис. 1 представлена общая схема системы производства. Использованы следующие обозначения:

$\mathrm{R}$ - возобновляемые ресурсы (прямая солнечная энергия, атмосфера, местные водные ресурсы);

$\mathrm{N}$ - невозобновляемые природные ресурсы (химические элементы из горных пород, природные источники энергии);

F - экономические ресурсы или экономические обратные связи системы, производственные силы. Эти экономические ресурсы делятся на:

M- денежные средства

$\mathrm{S}-$ услуги.

После разработки системной диаграммы были вычислены вклады всех компонент системы, заполнена исходная таблица (см. Таблица 2) для эмергетического анализа, которая включает количественные данные по всем входящим в процесс потокам, эмергию этих потоков, эмергию преобразования этих потоков в выходную продукцию, а также в остатки и потери.

Использовалась следующие данные по цементному заводу, полученные обработкой информации с официального сайта завода и сервиса Яндекс.Погода:

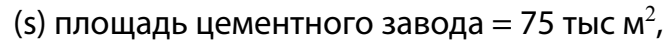

(r) количество осадков 0,563 м / год,

(u) количество солнечный дней 85,

(h) относительная влажность 72\%,

(w) скорость ветра 4 M/c, (g) скорость геострофического ветра $=4 / 0,7 \mathrm{~m} / \mathrm{c}$

(f) скорость стока 0,31 (\%/100),

(H) средняя высота над уровнем моря 27 м,

(i) инсоляция 1070 кВтч/ $\mathrm{m}^{2} /$ год=385,2Е+7 Дж/м²/год,

(а) альбедо 0,26, 
Таблица 2. Эмергетический баланс в цементном производстве в расчете на 1 день

\begin{tabular}{|c|c|c|c|c|c|}
\hline Ресурсы & $\begin{array}{l}\text { Формула для } \\
\text { величины потока }\end{array}$ & Единицы измерения & $\begin{array}{l}\text { Энергия всего } \\
\text { потока }\end{array}$ & $\begin{array}{l}\text { Затратность } \\
\text { эмДжДж }{ }^{-1}\end{array}$ & $\begin{array}{l}\text { Эмергия } \\
\text { эмДж/д }\end{array}$ \\
\hline R & & & & & $4,56 E+13$ \\
\hline Солнечный свет & $s \times u \times(1-a) / 365$ & Дж/д & $5,86 \mathrm{E}+11$ & 1 & $5,86 \mathrm{E}+11$ \\
\hline \multicolumn{6}{|l|}{ Дождь: } \\
\hline $\begin{array}{l}\text { Геопотенциальная } \\
\text { энергия }\end{array}$ & $\begin{array}{l}\mathrm{s} \times \mathrm{r} \times \mathrm{f} \times 1000 \mathrm{~K} / \mathrm{M}^{3} \times \\
\mathrm{H} \times 9,8 \mathrm{M} / \mathrm{c}^{2}\end{array}$ & Дж/д & $9,57 E+06$ & $1,31 E+04$ & $1,25 E+11$ \\
\hline $\begin{array}{l}\text { Химическая } \\
\text { потенцальная } \\
\text { энерги }\end{array}$ & $\begin{array}{l}s \times r \times e \times 1000 \mathrm{~K} / \mathrm{M}^{3} \\
\times \mathrm{G}\end{array}$ & Дж/д & $2,31 E+08$ & $2,35 E+04$ & $5,42 E+12$ \\
\hline Ветер & $s \times k \times g \times T$ & Дж/д & $1,56 \mathrm{E}+09$ & $1,90 \mathrm{E}+04$ & $2,96 \mathrm{E}+13$ \\
\hline $\begin{array}{l}\text { Геотермальное } \\
\text { тепло }\end{array}$ & $s \times t \times T$ & Дж/д & $2,27 E+08$ & 4,37 E+04 & $9,90 \mathrm{E}+12$ \\
\hline N & & & & & $7,77 \mathrm{E}+17$ \\
\hline Известняк & & кг/кг цемента & 0,95 & $1,27 E+12$ & $3,30 E+17$ \\
\hline Глина & & кг/кг цемента & 0,27 & $1,27 \mathrm{E}+12$ & $9,40 E+16$ \\
\hline Минер. добавки & & кг/кг цемента & 0,04 & $3,80 E+12$ & $4,16 E+16$ \\
\hline $\begin{array}{l}\text { Расход } \\
\text { теплоэнергии }\end{array}$ & & Дж/кг цемента & $2,52 E+06$ & $4,5 E+05$ & $3,11 \mathrm{E}+18$ \\
\hline$M, S$ & & & & & $1,03 E+17$ \\
\hline Себестоимость & & руб/кг цемента & 2,4 & $1,89 \mathrm{E}+10$ & $1,03 E+17$ \\
\hline Суммарная эмергия & & & & & $8,80 E+18$ \\
\hline
\end{tabular}

Таблица 3. Совокупные потоки эмергии в производственной системе цемента

\begin{tabular}{|l|l|}
\hline$R$ & $4,56 \mathrm{E}+13$ \\
\hline$N$ & $7,77 \mathrm{E}+17$ \\
\hline$I=R+N$ & $7,77 \mathrm{E}+17$ \\
\hline$F=M+S$ & $1,03 \mathrm{E}+17$ \\
\hline$Y=I+F$ & $8,80 \mathrm{E}+17$ \\
\hline
\end{tabular}

Таблица 4. Индексы эмергии для производственной системы цемента

\begin{tabular}{|l|l|l|}
\hline Коэффициент эмергетической отдачи & EYR=Y/F & $854 \%$ \\
\hline Коэффициент инвестирования эмергии & $\mathrm{EIR=F/I}$ & $13,3 \%$ \\
\hline Коэффициент экологической нагрузки & $\mathrm{ELR}=(\mathrm{F}+\mathrm{N}) / \mathrm{R}$ & $19300 \%$ \\
\hline Процент возобновляемости & $\% \mathrm{R}=\mathrm{R} / \mathrm{Y}$ & $0,005 \%$ \\
\hline $\begin{array}{l}\text { Коэффициент невозобновляемости местного } \\
\text { ресурса }\end{array}$ & $\% \mathrm{~N}=\mathrm{N} / \mathrm{Y}$ & $88 \%$ \\
\hline Коэффициент эмергетической устойчивости & $\mathrm{ESI}=\mathrm{EYR} / \mathrm{ELR}$ & $443 \%$ \\
\hline
\end{tabular}




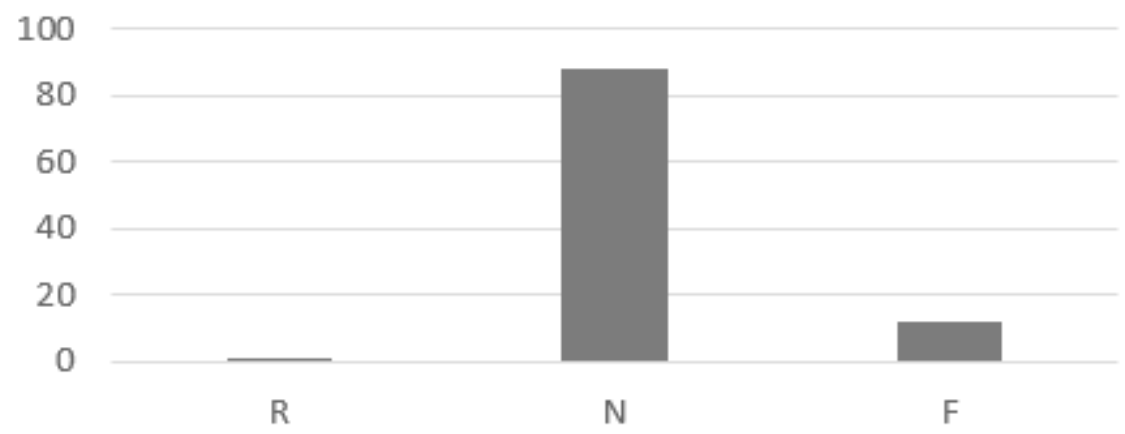

Рис. 2. Доли вкладов входящих потоков в общую эмергию

(Т) время 86400 c,

(G) - свободная химическая энергия Гиббса 4940 Дж/кг,

(е) скорость испарения 0,6 (\%/100),

(k) коэффициент сопротивления воздуха 0,001,

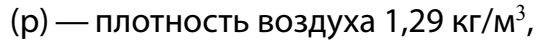

(t) геотермальный тепловой поток 0,035 Дж/ $\mathrm{m}^{2} / \mathrm{c}$

Состав портландцемента и расход электроэнергии рассчитывались в соответствии с [11]. Данные о затратности (transformity) взяты из [1, 2, 12]. Расчеты проводились по методике [5].

После получения значений индикаторов, показанных в Таблице 2, рассчитываются индексы для эмергетического анализа, приведенные выше в Таблице 1.

Сумма возобновляемых (R) и невозобновляемых (N) ресурсов дает общий вклад (I) в систему, I = R + N. Сумма вкладов природы (I) и экономических вкладов (F) обеспечивает общую эмергию (Y), которая заключается в рассматриваемой системе, $\mathrm{Y}=\mathrm{I}+\mathrm{F}$, что отображено в Таблице 3.

Согласно [5], эти индексы используются для анализа воздействия на окружающую среду и экономических условий промышленных систем (Таблица 4).

В результате анализа сделаны следующие выводы.

Коэффициент возобновляемости (R\%) составляет 0,005\%, что демонстрирует слабое поступление возобновляемой энергии для оцениваемой системы. Коэффициент невозобновляемости местного ресурса (N\%) показывает чрезмерные местные затраты ресурсов и значительное давление на местную среду. Относящийся к окружающей среде коэффициент нагрузки (ELR), иллюстрирует сильное давление на систему и необходимость мер снижения этой нагрузки. Коэффициент эмергетической отдачи (EYR) отражает конкурентоспособность завода. Индекс устойчивости Emergy (ESI) показывает устойчивый статус в долгосрочной перспективе.
Общий поток эмергии составлял 8,80E+17 эмДж. Наибольший вклад внесли невозобновимые природные ресурсы величиной.

Материальные ресурсы, которые использовались в производстве цемента, внесли наибольшую долю в используемую эмергию (88\% от общего количества). На долю материальных вложений и услуг приходилась вторая по величине доля эмергии (11\% от общего числа), что демонстрирует Рисунок 2.

Затратность может быть уменьшена за счет использования альтернативных добавок, но вычисление коэффициентов требует дополнительных исследований.

Когда потребляется больше невозобновляемых ресурсов, нагрузка на окружающую среду больше. Чрезмерные нагрузки на окружающую среду привносят серьезное ухудшение в экологические системы.

Моделирование показало, что уменьшение удельных затрат электроэнергии за счет обновления оборудования и использования минеральных добавок на 10\% снижает экологическую нагрузку на $2 \%$ и $8 \%$ соответственHO.

Качество входных потоков, локальное или внешнее их происхождение, возобновляемость или невозобновляемость, их больший или меньший спрос на экологическую поддержку делают продукт более или менее ценным в зависимости от того, какие движущие силы были вложены природой для того, чтобы сделать это и за сколько времени. То, что требует большой экологической нагрузки, не может быть лучшей ресурсной базой для устойчивости экономической системы. На основе анализа входных потоков энергий, каждый из которых характеризуется определенной затратностью и качеством, возможно разработать альтернативную систему или стратегию развития с гораздо лучшими результатами по сравнению с анализируемой. 


\section{ЛИТЕРАТУРА}

1. Odum, H.T.; Nilsson, P. O. Environmental accounting: emergy and environmental decision making. New York: John Wiley \& Sons, 1996.

2. Brown, M.T.; Ulgiati, S. Emergy-based indices and ratios to evaluate sustainability: monitoring economies and technology toward environmentally sound innovation. Ecological Engineering, v. 9, n. 1/2, p. 51-69, 1997.

3. E.L. Vitória, J.P. Rodrigues. Emergy efficiency analysis of sugarcane as a raw material for ethanol production. Revista Ciência Agronômica, v. 47, n. 4, p. 616-623, 2016.

4. Lanzotti, C.R.; Ortega, E.; Guerra, S.M.G. Emergy analysis and trends for ethanol production in Brazil. Emergy Synthesis, v.1, 2000.

5. Odum, H.T.; Brown, M.T.; Brandt-Williams, S. L. Handbook of emergy evaluation: a compendium of data for emergy computation issued in a series of folios: Folio\# 2: emergy of global processes. Gainesville: Center for Environmental Policy, University of Florida, 2000.

6. Zhang Wei, Wang Xiuhong, Zhang Hongye Emergy Based Sustainability Scenario Analysis of Oasis Agricultural Systems: A Case Study from Xinjiang, China // Journal of Resources and Ecology 2012 vol. 3 N1, pp. 026-032

7. Lulu Qu, Xueyi Shi, Chang Liu, Ye Yuan An Emergy-Based Hybrid Method for Assessing Sustainability of the Resource-Dependent Region // Sustainability 2017, 9, 153; doi:10.3390/su9010153

8. Xinyu Liu, Gengyuan L., Zhifeng Yang, Bin Chen, Sergio Ulgiati Comparing national environmental and economic performances through emergy sustainability indicators. Renewable and Sustainable Energy Reviews May 2016 D0l: 10.1016/j.rser.2015.12.188

9. Балтер Б.М., Фаминская М. В., Никитина Н. И., Бонкало Т. И., Романова Е. Ю., Ильгов В. И., Гарданова Ж. Р. Моделирование информационных потоков, связанных с глобализацией и влиянием индустриального развития на здоровье населения // Коллективная монография / Москва, 2020.

10. Балтер Б.М., Фаминская М. В., Никитина Н. И. О равновесии производственной и потребительской системы отсчетов применительно к загрязнению окружающей среды: эмергетический подход // В сборнике: Технические и естественные науки: проблемы, теория, практика. Сборник научных трудов по материалам Международной научно-практической конференции. Под общей редакцией Е. П. Ткачевой. 2019. С. $27-31$.

11. ГОСТ Р 56828.18-2017 Наилучшие доступные технологии. Производство цемента. Аспекты повышения энергетической эффективности.

12. Zhang, J.; S. Srinivasan, R.; Peng, C. A Systematic Approach to Calculate Unit Emergy Values of Cement Manufacturing in China Using Consumption Quota of Dry and Wet Raw Materials. Building 2020, 10, 128.

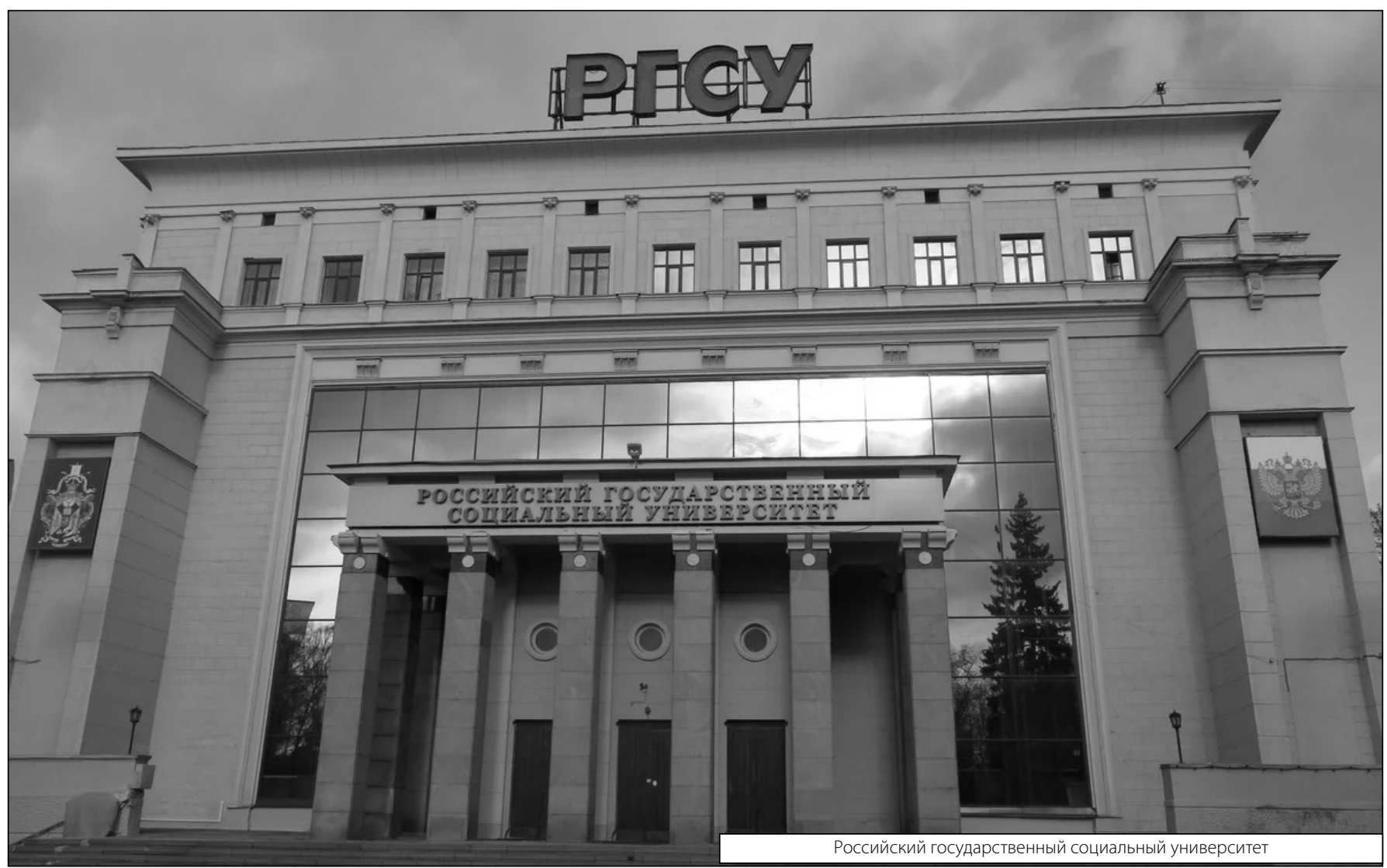

